\title{
REAL TIME SPECIAL EFFECTS GENERATION AND NOISE FILTRATION OF AUDIO SIGNAL USING MATLAB GUI
}

\author{
Saurabh R Prasad ${ }^{1}$, Pawan K. Gaikwad ${ }^{2}$ and Yashwant V Joshi ${ }^{3}$ \\ ${ }^{1}$ Department of Electronics and Telecommunication Engineering, DKTE Society's \\ Textile and Engineering Institute, Ichalkaranji, India \\ ${ }^{2}$ Department of Electronics, Shivaji University, Kolhapur, India and \\ ${ }^{3}$ Department of Electronics and Telecommunication Engineering, SGGS Institute of \\ Engineering and Technology, Nanded, India
}

\begin{abstract}
Digital signal processing is being increasingly used for audio processing applications. Digital audio effects refer to all those algorithms that are used for enhancing sound in any of the steps of a processing chain of music production. Real time audio effects generation is a highly challenging task in the field of signal processing. Now a day, almost every high end multimedia audio device does digital signal processing in one form or another. For years musicians have used different techniques to give their music a unique sound. Earlier, these techniques were implemented after a lot of work and experimentation. However, now with the emergence of digital signal processing this task is simplified to a great extent. In this article, the generations of special effects like echo, flanging, reverberation, stereo, karaoke, noise filtering etc are successfully implemented using MATLAB and an attractive GUI has been designed for the same.
\end{abstract}

\section{KEYWORDS}

Musical Signal Processing, Audio effects, Reverberation, Echo, Stereo Panning, Noise Filtration

\section{INTRODUCTION}

Signal Processing is such a broad field that it almost entirely covers electronics. It can be classified in two main categories, analog signal processing and digital signal processing. The analog signal processing is a forerunner amongst these two types. Even though there are several drawbacks of the former, still it enjoys the fact that all real world signals are analog in nature, thus there is no need to convert signal from analog domain to digital domain and vice-a-versa. However, in digital signal processing, we need ADC and DAC as additional components. ADC is used to convert real world analog signal into digital so that it can be applied as input to DSP processor; and DAC is used to convert processed digital signal to analog form in order to present to real world. Thus in digital signal processing, the input signal to DSP processor is one sequence of numbers, and it's output is another sequence of numbers.

The DSP processors process the input sequence of numbers, without caring whether the input is audio signal, video signal, an image, multimedia or something else. Thus digital signal processing is the numerical manipulation of signals. It can perform a variety of operations on signal such as measurement, filtration, amplification, attenuation, delay, compression, or signal generation itself. 
Any type of analog signal such as discrete time, discrete frequency, or other discrete domain signals can be conveniently represented as digital signals in the form of a sequence of numbers to permit the digital processing. The digital signal processing renders computational power of microprocessors to signal processing and thus demonstrates many advantages over analog processing. It's applications are uncountable, and few of them are error detection and correction in data transmission, data compression, digital audio effect generation, audio-video mixing etc. The DSP processors have become highly popular owing to its different advantages like costeffectiveness, speed, energy efficiency etc. The distinguishing feature of DSP over it's analog counterpart is capability of software control; i.e. keeping the same digital hardware but different software program, varied applications are possible. In addition DSP processors are field programmable. The inherent disadvantage of DSP is quantization error which is irrecoverable; however, this can be reduced to a greater extent using more number of bits per sample, or in other words by decreasing quantization step size.

\subsection{General Applications of DSP}

Digital signal processing is one of the core technologies in rapidly growing application areas such as audio and speech processing, sonar and radar signal processing, antenna beam-forming, spectral estimation, statistical signal processing, digital image processing, communications, control, data compression, financial signal processing, biomedical, seismic data processing, and many others. Specific consumer grade examples of applications of DSP include mobile phones for speech compression and transmission, Hi-fi sound system for Digital Room Correction (DRC) and audio effects generation, Sound Reinforcement System, Medical imaging such as MRI, and Computer graphics etc.

There is increasing trend in number and variety of products that use DSP processors or perform some sort of digital signal processing. DSP is a key component in many products used in varied fields such as medicine, communication, industry, or consumer market which implement some sort of signal processing using a wide variety of processors such as general purpose microprocessors, Microcontroller, Field Programmable Gate Arrays (FPGAs), Custom ASICs etc. Due to increasing popularity of the above mentioned applications, a variety of the DSP-enabled processors has expanded greatly. Digital Signal Processing operations such as convolution, autocorrelation, cross correlation, filtering etc require the three basic operations; multiplication, addition and delay repeatedly. So, DSP processors are the specialized processors that can perform these three operations at a faster rate. Thus DSPs processors are specifically designed for highspeed numeric processing applications and its hardware architecture as well as instruction sets are optimized for the same. This is an essential requirement for processing digital data and representing analog signals in real time.

DSP algorithms can run on standard computers, on specialized processors called digital signal processors, and on specialized task oriented hardware known as Application-specific integrated circuit (ASICs). In addition, now days other technologies used for digital signal processing are emerging that include field-programmable gate arrays (FPGAs), powerful general purpose microprocessors, and digital signal controllers etc. The last one is used mostly for industrial control applications such as motor control. Often non real-time applications use general purpose computer in order to perform signal processing tasks with economy. Digital Signal Processing is similar to any other data processing except it uses specialized DSP mathematical techniques such as convolution and FFT. In digital signal processing, a continuous time signal is discretized by uniform sampling carried out in time domain or space domain. The most common software doing this kind of digital signal processing is 'Photoshop'. However, when the application requirement is real-time, DSP is often implemented using specialized DSP processors such as DSP6000 
family's TMS320 processor or the SHARC. These processors are of two types, fixed-point arithmetic, and floating point arithmetic. The later one is more common in powerful versions of DSP processors. For faster applications design and development FPGAs might be used. Now days multi-core implementations of DSPs have started to emerge. For volume production and faster applications, ASICs might be designed specifically. For slow applications, a traditional processor such as a microcontroller with DSP enriched features may be useful.

\subsection{Glimpses of Audio Effect and Its Historical Development}

Audio is a one dimensional signal, i.e. physical quantity varies with only one independent variable, time. Therefore in digital domain the audio is represented by vectors. Audio effects are artificially created or enhanced in order to emphasize artistic or other content of multimedia such as music, video games, motion picture, television, animation etc, and also live performance. Most of special effects are generated with three basic operations to signal viz. delay, scalar multiplication, and addition. It can be noted that such operations are nothing but a sort of filtering of audio signal.

Delay is an integral part of each and every audio effect. A unit delay element stores the incoming sample stream for one sample period, and then release the output sample stream. The delayed signal may either be played back for one time or for multiple times to create the desired effect. The duration of delay and number of delay feedbacks changes the audio effect taking place. The delay unit in audio special effects generation is such an important task that the chronological development of this process is thoroughly discussed in following section.

\subsubsection{Analog delay}

In old days when neither analog nor digital audio technologies were available, music employing a delayed echo had to be recorded in a naturally reverberant room. However, this caused great inconvenience to both musicians and sound engineers. This problem was an inspiration to discover a machine that could synthesize echoes of desired interval or amplitude. The earlier such systems were analog in nature.

\subsubsection{Early delay systems}

The first delay effect generation is identified as "tape based delay" and it used magnetic tape as recording medium. Here, the duration of delay could be controlled by adjusting the length of the loop of tape. This technique was most popular among early composers such as Pierre Schaeffer those adopted 'Musique concrete'; an experimental technique of musical composition using recorded sounds as raw material. The great composers of $20^{\text {th }}$ century such as Karlheinz Stockhausen devised a complex system that incorporated the use of long tapes with multiple recorders and were used to process the input of a live performer. Audio engineers quickly adapted these techniques and also invented similar techniques known as 'Plate Reverb' which used an electromechanical transducer designed to simulate natural echo.

In the late 1940s and early 1950s, sound engineers prepared themselves to invent new devices those were applicable in recording studios as well as in live performance. One step ahead task was the creation of electric guitar invented by Les Paul. It is assumed that this invention could make the sound of rock and roll possible. He successfully experimented for generating tape delay, phasing and multi-track recording those caused widespread attention. Ray Butts created a landmark device 'EchoSonic', which was a portable guitar amplifier with a built-in tape echo which was widely used in country music by American guitarist named Chet Atkins [1]. Tape 
echoes became commercially available in the 1950s. An 'echo machine' was the early name for a sound processing machine that used electronic instruments to repeat the sound and produce a simulated echo. This device could become much popular and used by contemporary renowned guitarists Brian May, Jimmy Page and Syd Barrett among others. One more analog delay device was named 'Echoplex' which used a tape loop [2]. In this device the length of delay could be adjusted by changing the distance between the record and playback head. Another similar device Roland's RE-201, commonly known as 'Space Echo' was produced by the Roland Corporation in 1973. This device consisted of a record head and multiple playback heads with variable tape speed and used for analog audio delay effects generation. In this device the time between successive echoes were adjusted by varying the tape speed [3] and the duration of echo was adjusted by changing the amount of echo signal fed back into the pre-echo signal.

\subsubsection{Solid state}

Another alternative to tape loop delay was solid state delay that used a bucket brigade and so called as bucket-brigade device (BBD) [4]. It was devised in 1969 by F. Sangster and K. Teer of the Philips Research Labs and was nothing but a discrete-time analog delay line. Its name originates from analogy with the term 'bucket brigade' that is used for a line of people passing buckets of water. Similarly, it consisted of a series of capacitors $\mathrm{C}_{0}$ to $\mathrm{C}_{\mathrm{n}}$. The stored analog signal was moved along the line of capacitors, one step at each clock cycle. Solid state analog bucket brigade delay circuits became available from 1970s. The commercially available BBD was made available from Reticon Corporation in 1980s. The miniature of BBD was a celebrated integrated circuit 'SAD-1024' that implemented two 512-stage analog delay lines available in 16pin DIP packaging [5]. This chip and design were used in well known Rockman amplifier. In fact solid state analog delays are less flexible than digital delays and also they generally have a shorter delay time, still several devices such as the 'Boss DM-2' are used even today for their natural echo quality and progressively decaying echoes.

\subsubsection{Digital delay}

The easy availability of inexpensive digital electronics circuits and devices in the late 1970s and 1980 s led to the development of digital delay generation devices. Initially, they were available in the form of expensive rack-mounted units but later as costs came down and the electronics was miniaturized, they could became available in the form of foot pedals [6]. The first digital delay offered in a pedal was the Boss DD-2 in 1984. Digital delay generation machines sample the continuous time signal and pass through a series of digital signal processors that record it into a storage buffer. Finally play back of the stored audio samples takes place based on parameters set by the user. The delayed output may be mixed with the unmodified signal either in the form of feedback or feed forward and finally applied to digital-to-analog converter for output.

\subsubsection{Software}

The next step to digital delay system was invention of software-based delay systems. Software delays enjoy the advantage of greater flexibility over most recent digital hardware delays. Software delays use system memory available on personal computers enabling practically limitless storage for the audio buffer. The natural efficiency of software delay algorithms has made the delay implementation unparallel compared to any other older technologies. Therefore, software-based delays are most popular among musicians in electronic genres. Now days audition usually takes place in computerized software controlled digital audio editing and mixing environment. 


\subsection{Audio effects using DSP}

Real time audio special effect generation is an important and one of challenging tasks. This is an inevitable and integral part of any sound casting industry. The requirement of real time design with feasibility has caused a widespread popularity of different digital audio effect generation algorithms. With earlier techniques it was difficult to implement different audio effects. However, invention and popularization of digital signal processing has made the task of audio effects implementation pretty simple compared to analog design. Special audio effects are used by a variety of sound casting industries like film industry, television, radio, public address system etc. MATLAB is a popular tool which is highly and universally used in the field of signal processing. Simulink, developed by MathWorks, provides an easier block diagram based graphical programming environment. Simulink can be used for modeling and simulating physical systems. The Simulink model is designed without any prior calculation of model parameters thus the model design is supported by trial and error methodology [7-8].

\section{THEORY OF GENERATED AUDIO EFFECTS WITH RESULT}

This paper focuses on the theory, design, and implementation of desired effects to input audio signal. MATLAB based design approach help the sound engineer to easily configure desired audio effects by varying the values of predefined Simulink blocks, or GUI parameters. Simulink or GUI parameters shape and control the transfer function for the desired effects. Instead of doing mathematical formulation, the parameters can be set with trial and error and with fine tuning, excellent audio effects are observed. An attractive GUI has been designed for some of the effects [9]. The DSP Techniques discussed here can be used to implement many of the features found in digital mixing consoles and digital recorders. Some examples for various effects that were implemented using MATLAB platform are presented here.

\subsection{Echo}

Acoustic echo occurs when an audio signal is reflected by different surfaces such as wall, ceiling etc in a real environment, resulting in the original signal plus attenuated time delayed images of this signal. Thus, to artificially generate the effect of echo in a large hall, one or several delayed signals are added to the original signal. It is estimated that to be perceived as distinct echo, the delay interval has to be higher than 35 milliseconds [10]. A Simulink model has been designed as shown in figure no 1 which comprises of three main blocks; delay, gain, and sum. Two additional blocks are also present in the figure, one 'From Wave File' block for applying input to the model and other 'To Audio Device' block to listen the processed output. From trial and error the gain and delay parameters are set to get the desired amount of echo. Figure no 2 shows Simulink Parameter setting for delay block. The change in the sound for different values of delays ranging from 100 to 20000 is varied for optimal adjustment. Similarly figure no 3 graphically indicates the original signal and echo signal. 
International Journal on Computational Science \& Applications (IJCSA) Vol.5,No.6, December 2015

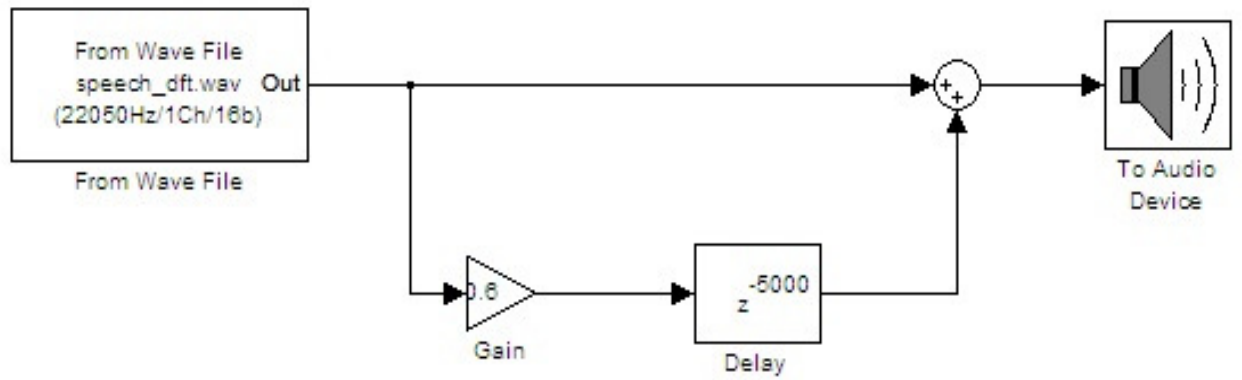

Figure 1.Simulink Design for Echo Effect

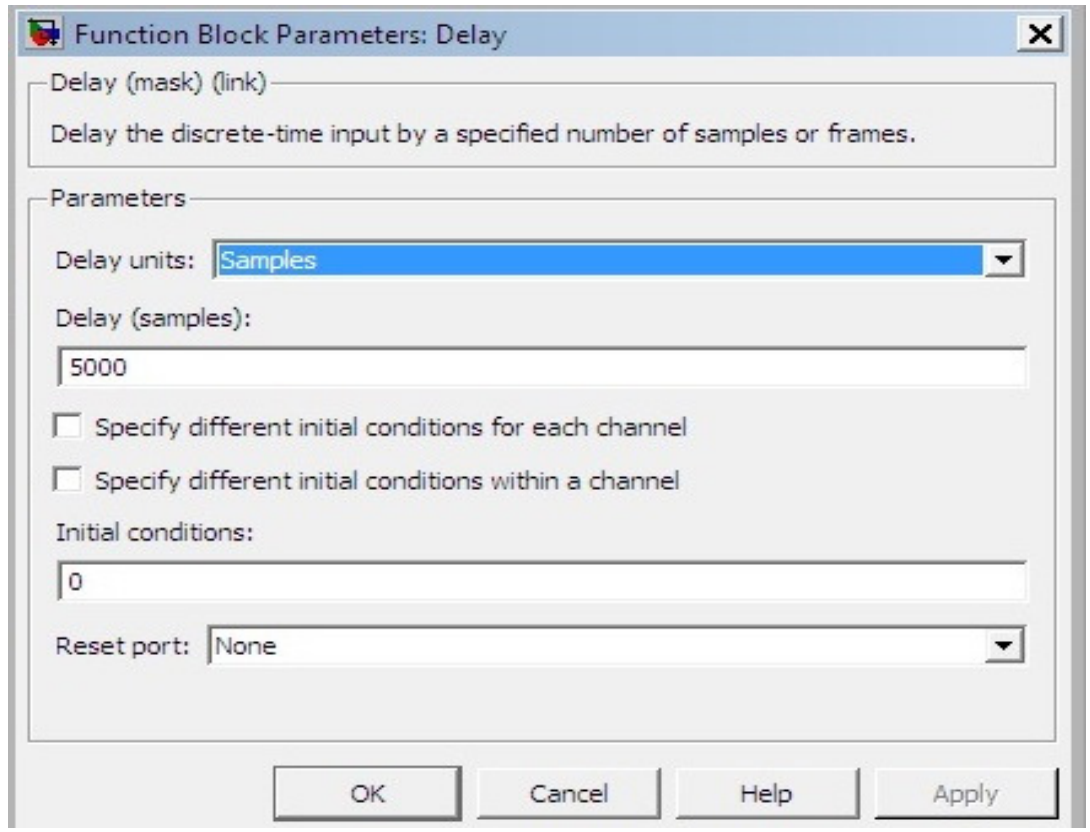

Figure 2. Setting Simulink Parameters for Delay Block of Simulink Model 

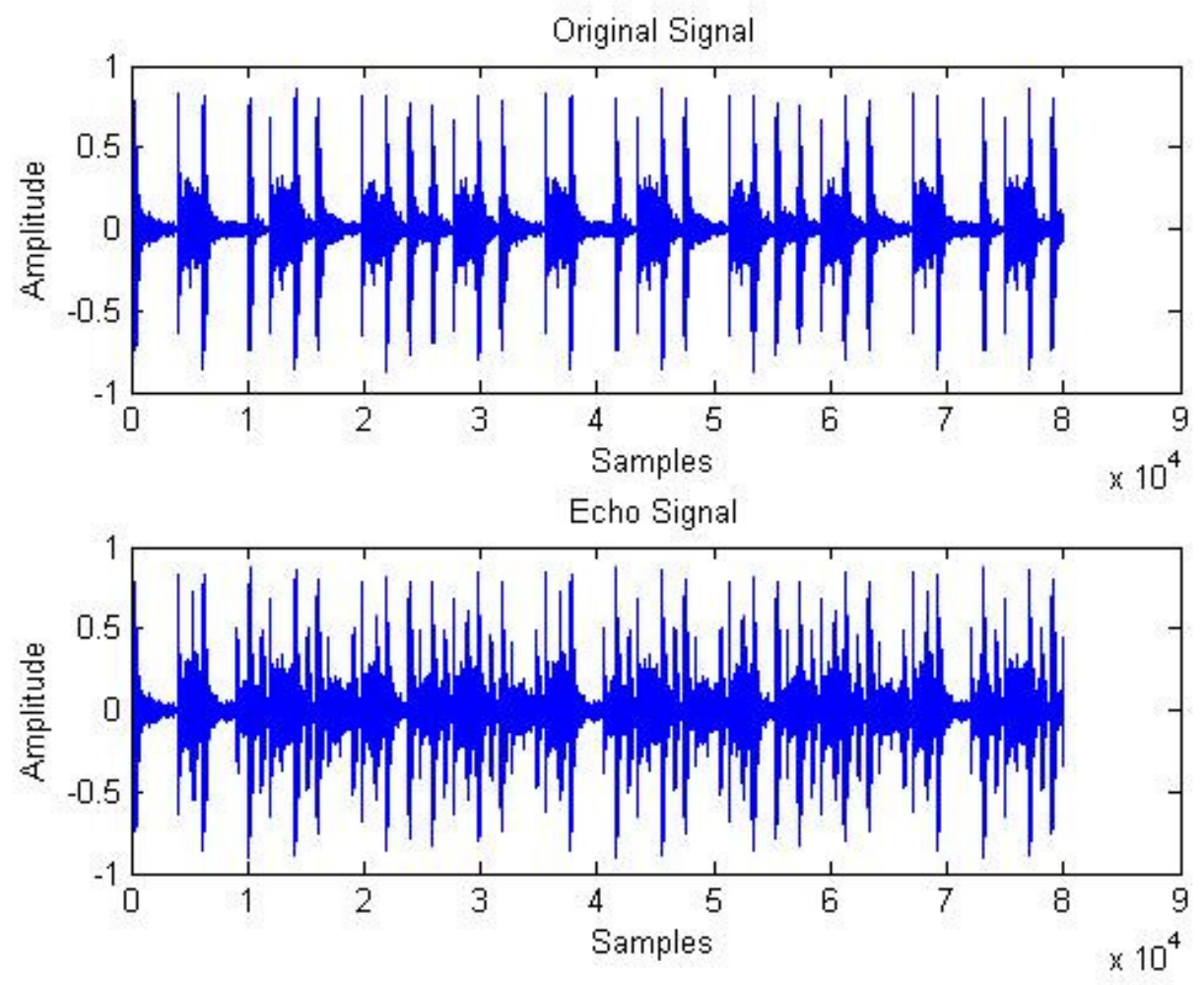

Figure 3. Plotting Original and Echo Signal using MATLAB

\subsection{Reverberation}

Similar to echo, reverberation also occurs due to reflections of sound, as shown in figure no 4 . Reverberation is defined as a phenomenon of persistence of sound when the source has ceased to produce it. A reverberation, also called reverb, is created when a sound is reflected from multiple reflecting surfaces causing a large number of reflections to build up. This is followed by progressive decay as the sound is absorbed by the different absorbing surfaces such as furniture, audience, curtains etc. This is most noticeable when the source of sound stops but the reflections persist and continuously decreasing in amplitude until reaching zero amplitude [11]. When reverberation exists for several seconds, it appears that the sound is presented in a large room and it gives a desired effect.

In the acoustic design of a room or auditorium the correct reverberation time is calculated using Sabine's formula. The calculated value of reverberation time is practically achieved using suitable absorbents, or openings. For example; lecture room, orchestra, industrial house, temple etc have different values of reverberation time that need to be maintained. But many times the correct reverberation time is neither available at studio side nor at playback side. But using DSP this effect can be artificially simulated using the techniques of audio effect generation. In a closed space, such as a musical concert, the sound reaching the listener comprises of three different components namely direct audio, early reflection and reverberation. The direct sound is always perceived by the listener's ear first because of shortest path, followed by the reverberant sound. The time span between them is called as 'pre-delay'. The reverberation is composed of densely packed echoes. It is estimated that approximately 1000 echoes per second are required to create a 
reverberation that sounds free of flutter. In comparison to a distinct echo that needs a delay of above $35 \mathrm{~ms}$ between successive reflections, the delay required here is less than $35 \mathrm{~ms}$.

Reverberation is a standard audio effect used universally in digital audio workstations (DAWs). A simple and effective way of artificially generating reverberation is as shown in figure no 5. This figure represents a Simulink model with three different blocks namely gain, delay and adder configured in feedback loop with proper parameter setting. The parameters of block can be modified and the resulting sound from speaker can be observed. Thus, the gain and delay block parameters are set by trial and error and general intuition. The setting of gain block parameter is as shown in figure no 6 . The feedback loop is forms first order IIR filter. The different types of reverberation effect generation those were used in past period as well as new techniques used today are discussed in following section.

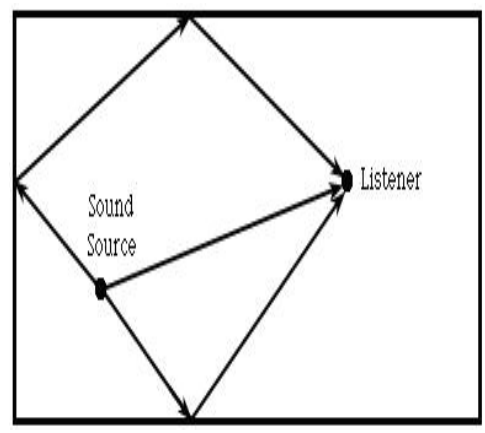

Figure 4. Generation of Echo and Reverberation

\subsubsection{Chamber Reverberator}

Before invention of digital signal processing technology, the reverberation effect was created using physical space in the form of natural echo chamber. In this method, a loudspeaker would play the sound, and then a microphone would pick it up again, and there was a long chain of this loudspeaker-microphone pair which could generate this effect. This technique is used in practice even today but due to disadvantages like necessity of a dedicated soundproofed room and the associated problem of difficulty in varying the reverberation time, it is not widely used [12].

\subsubsection{Digital Reverberator}

Digital reverberator use various signal processing algorithms for creating the desired reverberation effect. Since reverberation is caused by mixture of a large number of echoes, reverberation algorithms uses several feedback delays to create a large number of decaying echoes [13]. Now days the audio technology is advanced to such an extent that the high end digital reverberator can simulate the time and frequency domain response of a specific room as per our need by providing the inputs of room dimensions, absorption and other properties [14].

\subsubsection{Convolution reverberator}

Convolution is a mathematical operation and building block of most of digital signal processing or filtering operations. Convolution reverberation is a process used for digitally simulating reverberation in which out of two signals involved in convolution operation, one is a pre-recorded impulse response of the space being modeled, and another is sound to be echoed. In this method, 
the impulse response samples are stored in a digital signal processing system and then convolved with the incoming audio signal to be processed [15].

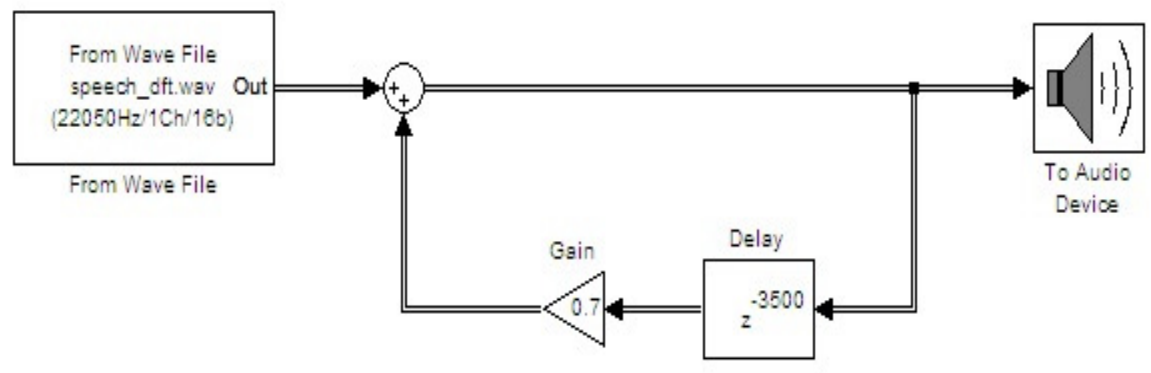

Figure 5. Simulink Model Design for Reverberation Effect

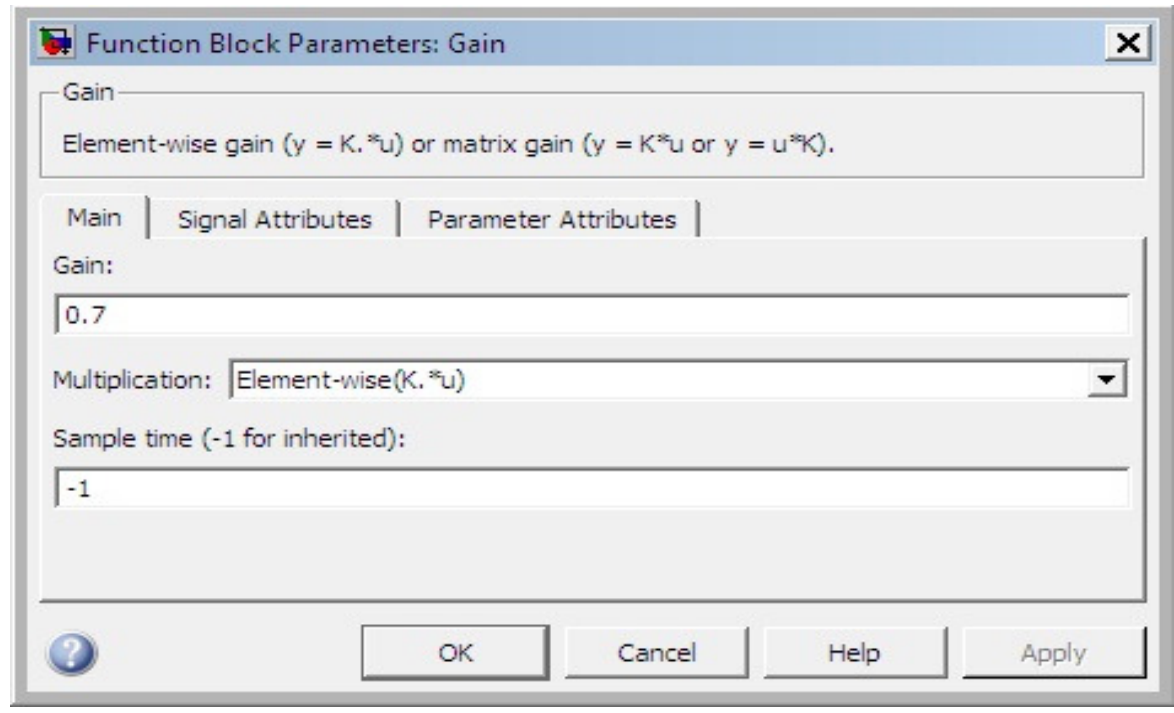

Figure 6. Setting Gain block parameter of reverberation model

\subsection{Flanger}

Flanging effect also known as "whooshing sound" is a type of phasing effect. To create Flanging effect, a delayed signal is added to the original signal with a continuously variable delay of duration smaller than about $10 \mathrm{~ms}$. This effect is now done electronically using DSP, but in old days this effect was artificially generated by means of playing the same recording on two different but synchronized tape players and then combining those signals together. As long as the two players were synchronized, the result of mixing would sound almost normal, but when the sound casting operator placed his/her finger on the flange of one of the players (hence named "flanger"), that player would slow down and its signal would go out-of-phase w.r.t. another one, producing a phasing effect. Once the operator took off his finger, the player would speed up again until finally it could become in phase with the master player. This was performed rhythmically and created flanging effect. This effect sounds to slide up the frequency spectrum of the audio 
signal [16].The audio flanging effect generation using Simulink and GUI has been shown in figure no 7 and 8 resp. Similarly this effect is listened from speaker sound.

Phaser is another way of creating similar effect. Here the audio signal is split in two parts. One part is applied to an all-pass filter that causes a phase-shift and then this part is mixed with other unfiltered part of the audio signal. The phaser effect was originally a simpler implementation of the flanger effect since delays were difficult to implement with analog equipments [17]. The phasers artificially enhance the natural sounds such as human voice. Similarly, when a delayed signal is added to the original signal with a constant delay then chorus effect is observed. The delay has to be short so as not to be perceived as an echo. However, if the delay is too short, it will destructively interfere with the un-delayed signal and create a flanging effect. The standard delay time for generating chorus effect is from about $5 \mathrm{~ms}$ to $40 \mathrm{~ms}$ [18].

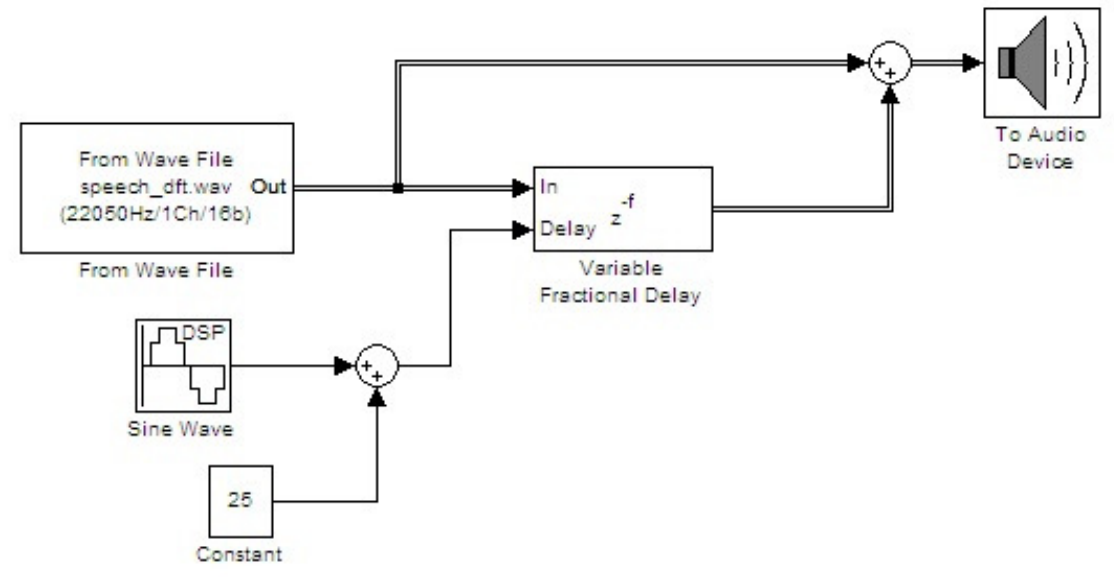

Figure 7. Simulink Model Design for Flanging Effect

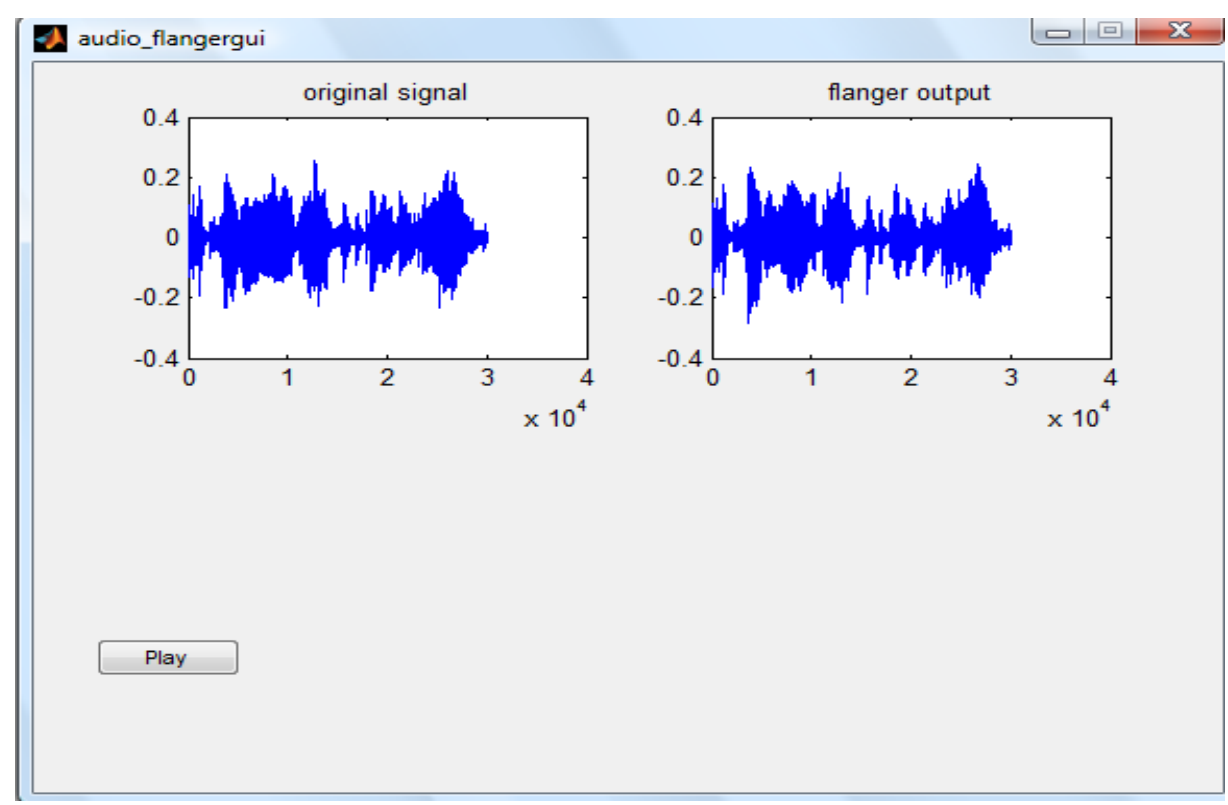

Figure 8. Audio Flanging Effect Using MATLAB GUI 


\subsection{Stereo/ Panner}

One way of creating the feeling of stereo for monophonic recording is to add a delayed version of the signal to itself. In monophonic sound there is no directional effect. On contrary in stereo sound directional effect is present thus audience can judge the direction from which sound is heard, i.e. left or right direction. Similarly in quadrophony, there are four directions of sound giving more natural effects. Thus stereophonic sound is preferred over monophonic. Similarly, Panning is the distribution of a sound signal into a new stereo sound track determined by a pan control setting. A stereo panner has an internal architecture which determines how much of a source signal is sent to the left and right speaker. This signal distribution is also called a taper. Thus, panning is analogous to stereo effect generation in which directional effect is given to the sound [19]. The implementation of stereo panning is done using Simulink and MATLAB GUI and shown in fig no 9 to 11 .

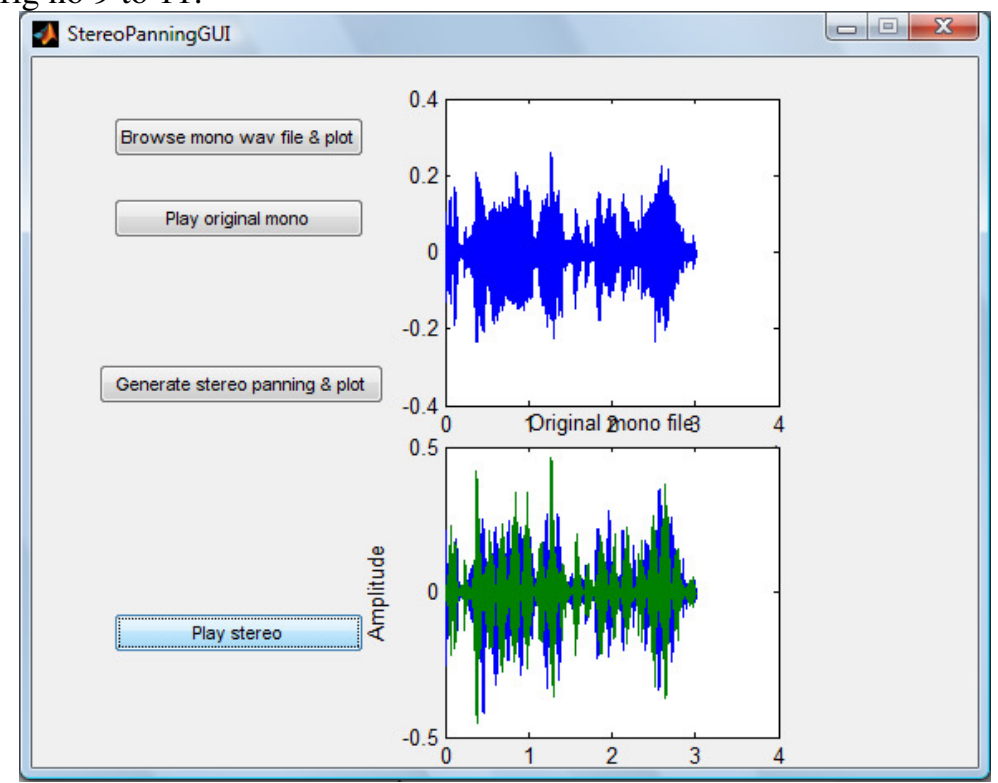

Figure 9. Stereo Effect using MATLAB GUI 
International Journal on Computational Science \& Applications (IJCSA) Vol.5,No.6, December 2015

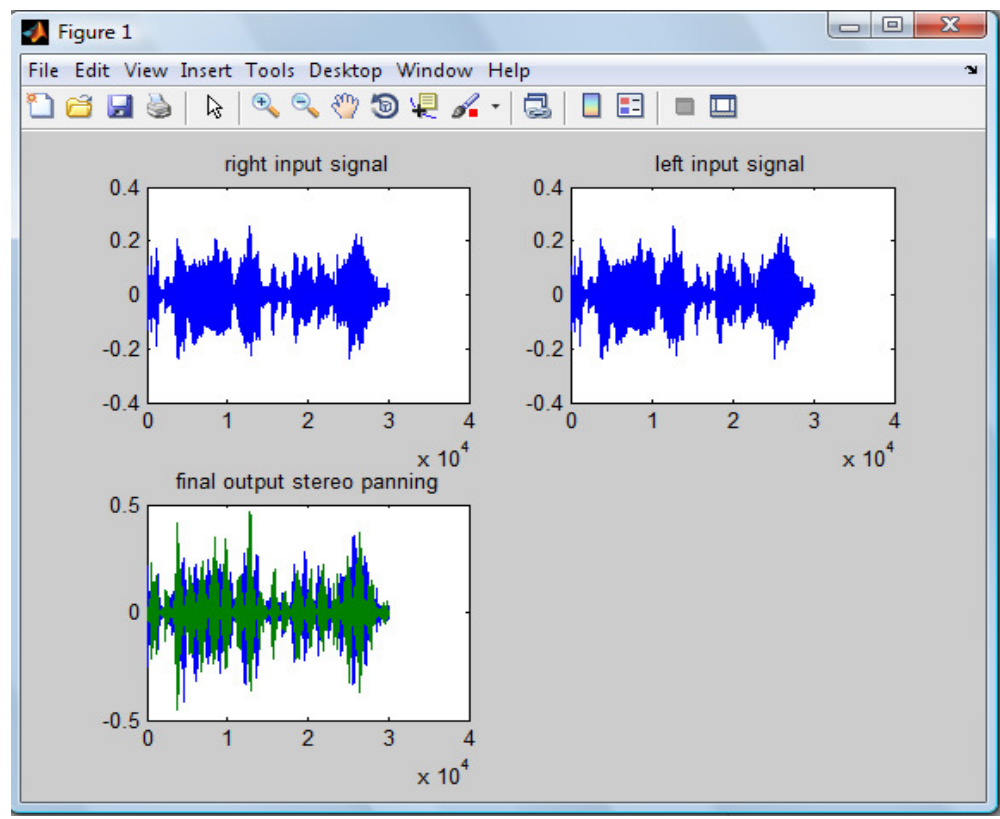

Figure 10. Stereo Panning showing left, right and combined signal

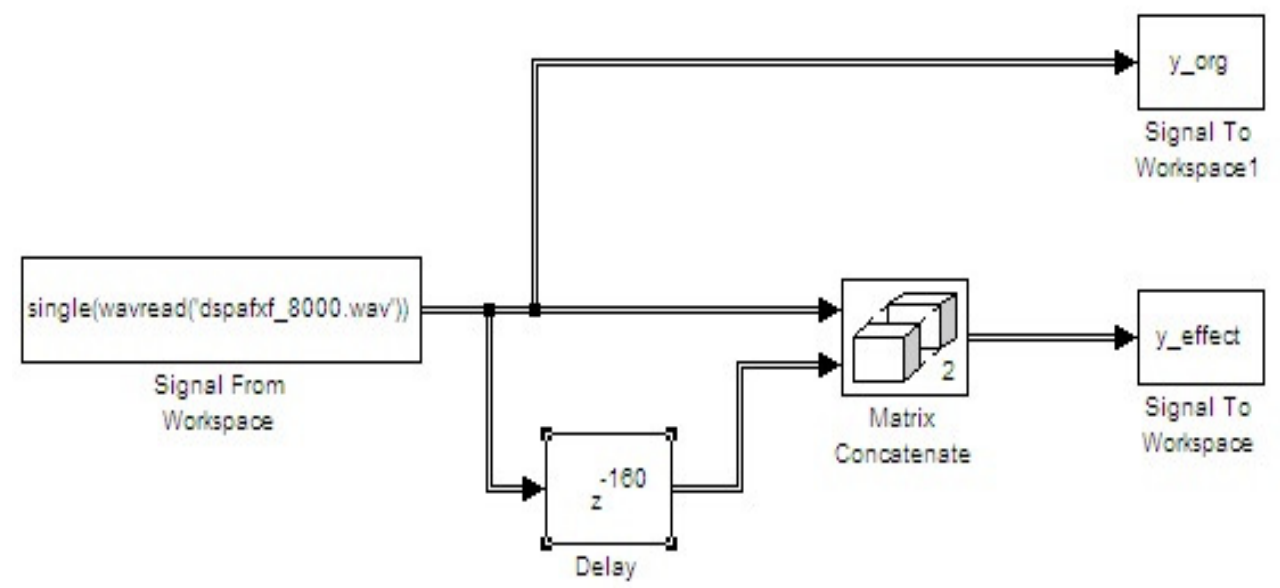

Figure 11. Simulink Model for Stereo Effect Generation

\subsection{Karaoke Effect}

Karaoke is a term used by recording engineers to refer "empty track" meaning there is no vocal track. It is a form of interactive entertainment in which an amateur singer's singing is supported by 'music only' recording. The recording is usually a well-known popular song minus vocal track. In sophisticated system, lyrics are usually displayed on a video screen, along with some sort of guide to the singer in the form of some moving graphic symbol, changing light etc. A karaoke box is also abbreviated as KTV [20]. The MATLAB GUI implementation of Karaoke effect is as shown in fig. no 12 . 


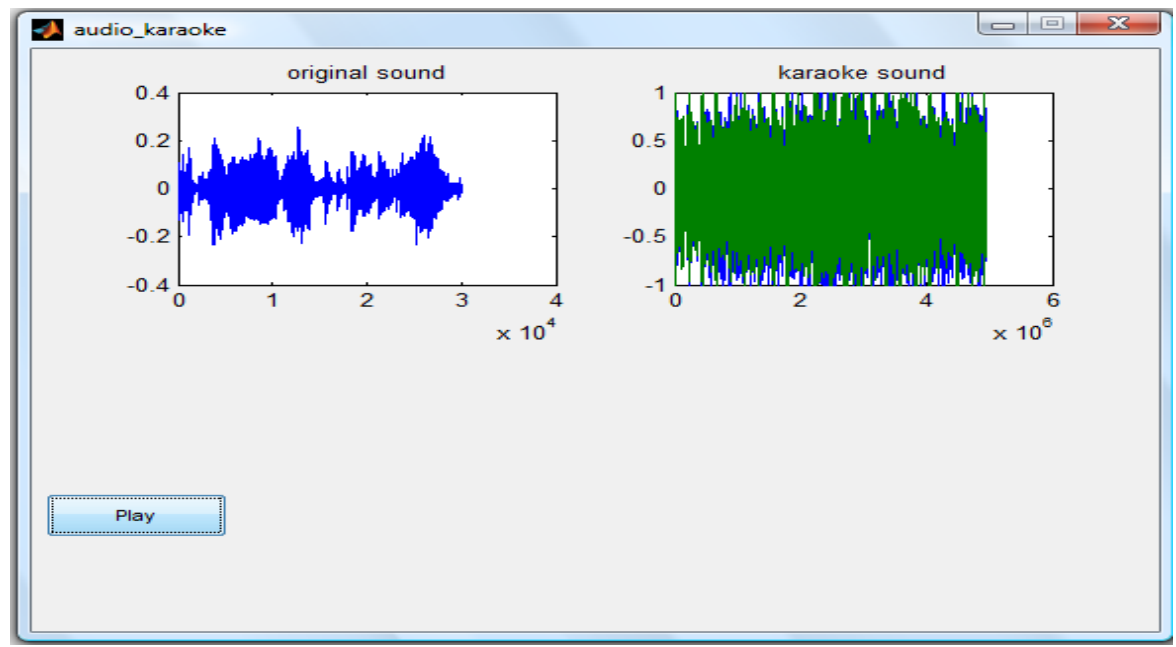

Figure 12. Karaoke Effect using MATLAB GUI

\section{FILTERING}

Noise filtering of audio signal is an extremely important task in audio enhancement. The noise filtering in DSP is usually carried out by two methods namely fixed FIR or IIR filter, and the adaptive Filter. Though fixed filters are highly used in practice, still the advantages of adaptive filter outweighs over fixed filter so here adaptive filter is used for noise filtering application. An adaptive filter is a filter which self adjusts its transfer function according to an optimizing algorithm in order to produce the desired result. There are two popular adaptive filtering algorithms, LMS and RLS. The former one offers an easy, simpler, and robust implementation, so used here.

\subsection{Algorithm}

The fixed filters are suitable when signal and noise both are stationary signal and occupy different frequency bands. But when they lie in same frequency band, or their characteristics are changing then the use of adaptive filters is inevitable. If there is change in input signal or change in channel characteristics, the adaptive filter adapts itself to produce the desired optimum output. The optimization is done with respect to minimization of some function, called as cost function, of error signal. LMS algorithm uses mean square error (MSE) as the cost function, where the error signal is difference between the desired signal and the filter output signal. Thus adaptive filter accepts two inputs those are desired signal and input signal and it produces two outputs viz. filter output and error signal. The filter output is the convolution of filter coefficients with the input signal.

This example illustrates the use of LMS algorithm in adaptive FIR filter design to filter the signal corrupted with noise in a music recording. There are two inputs; the first input is the filtered noise $\mathrm{x}(\mathrm{n})$ and the second input called as the desired signal $\mathrm{d}(\mathrm{n})$ is the music with unfiltered noise. The LMS algorithm computes the filtered output $y(n)$ which is convolution between $x(n)$ and filter eight $\mathrm{w}(\mathrm{n})$. A subtractor is used to subtract the filter output from desired signal to produce the error signal e(n) that is applied to algorithm. The algorithm in its next iteration computes the filter weight vector $w(n+1)$ to minimize the mean square error of $e(n)[21]$. When the simulation is 
started, we hear both the noise and the music. Over time, the adaptive filter removes the noise and we hear only the clean music. Figure no 13 and 14 resp. shows Simulink model for LMS Adaptive Noise Filter and corresponding simulation result.

\subsection{Filtering Steps}

The filtering takes place in three phases:

- Convolution

The convolution operation between instantaneous filter weight vector with input signal vector occurs in order to filter noise and produce an estimate of the noise content in the desired signal.

- Error Computation

The instantaneous error $e(n)$ is the difference between the desired signal $d(n)$ and the filter output $y(n)$. The adaptive filter tries to minimize some function of error. This function is called as cost function and LMS algorithm uses Mean square error (MSE) as the cost function. In noise cancellation application, error signal represents noise free filtered audio signal.

- Weight update

To minimize the cost function, adaptive filter iteratively searches for negative gradient and update the weight or filter coefficients accordingly. The new value of the filter weights is the old value of the filter weights plus a correction factor that is based on the error signal, the distorted signal, and the adaptation step size. The weight update equation of LMS algorithm is given by, $w(n+1)=w(n)+2 \mu e(n) x(n)$ where $\mu$ is the step size parameter. The step size is supposed to lie in between the lower bound of 0 and upper bound of $1 / E\left[x^{2}(n)\right]$. 
International Journal on Computational Science \& Applications (IJCSA) Vol.5,No.6, December 2015

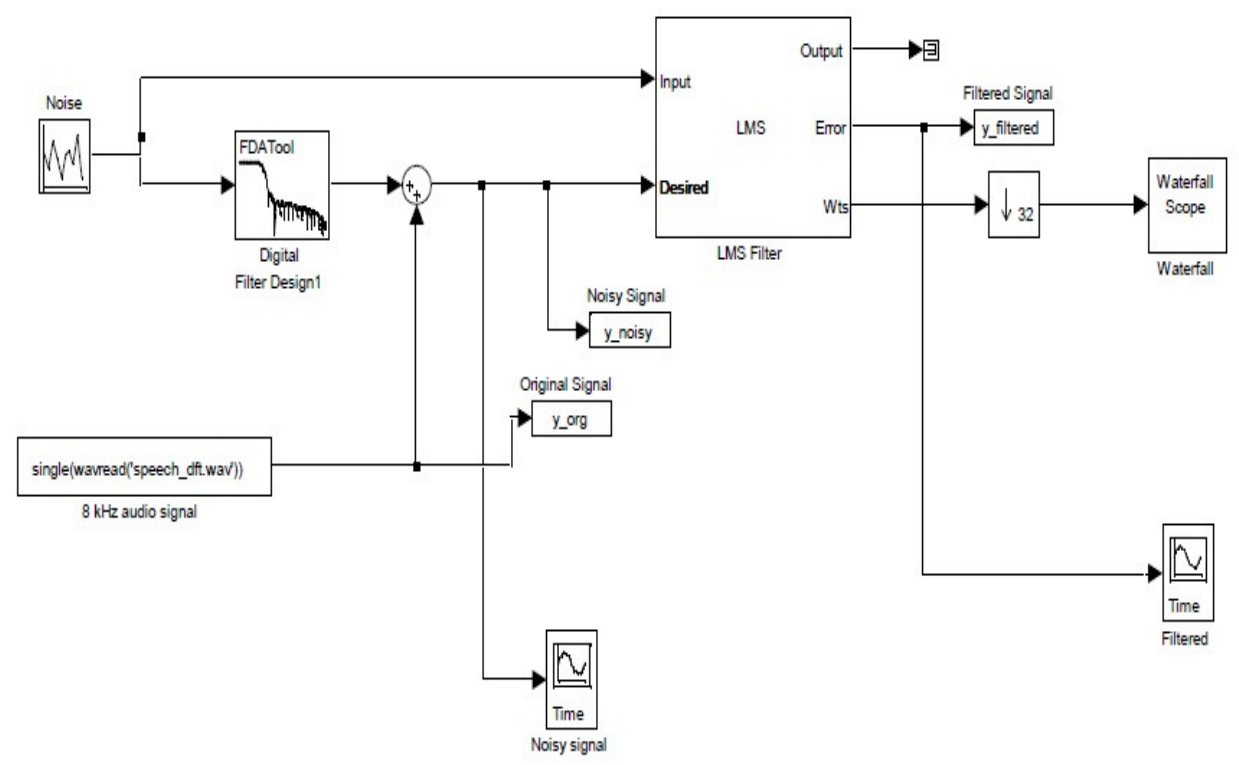

Figure 13. Simulink Model for Noise Filtering Using LMS Algorithm
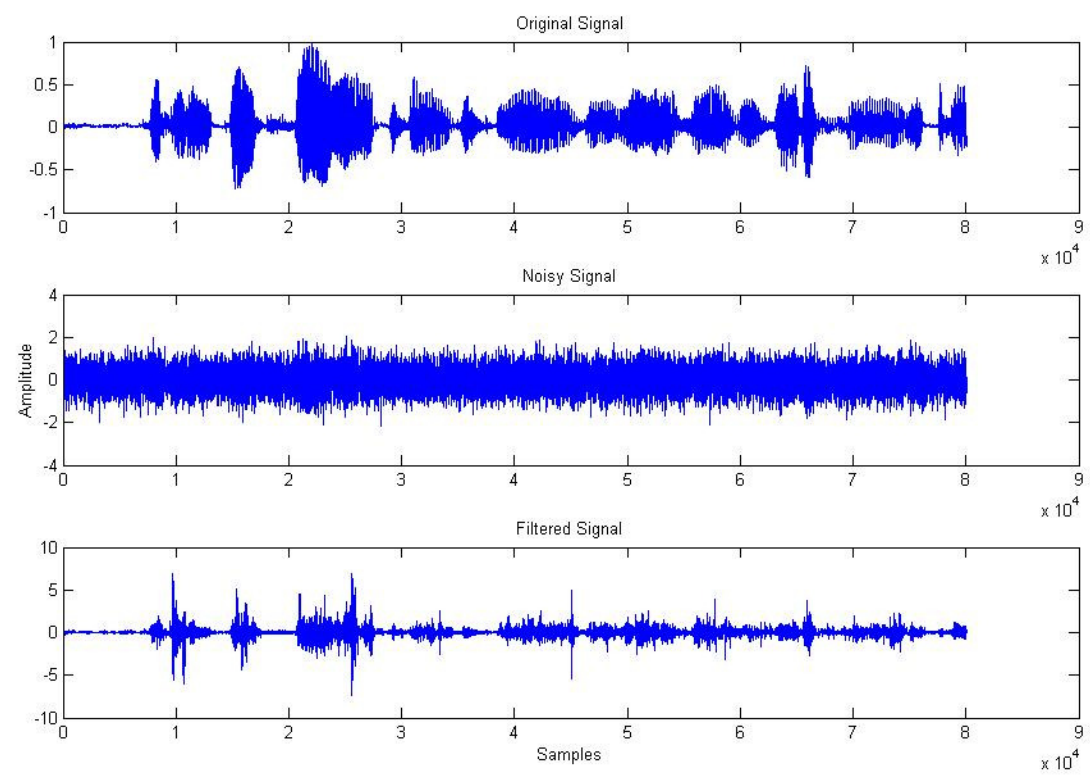

Figure 14. LMS Adaptive Filtering output showing Original Signal, Noisy Signal, and Filtered Signal

\section{CONCLUSION}

Audio effect generation using Digital Signal Processing is very simple, easy to implement, and less time consuming task compared to its analog counterpart that required a lot of time to develop desired effect. The implementation of these audio effects can be done using standard Personal 
Computer, dedicated DSP hardware; specially designed ASIC, High end DSP enabled microcontroller or FPGAs. In this research work, all the effects have been implemented using PC with MATLAB software. Different audio effects such as echo, reverberation, flanging, phasing, stereo panning, karaoke, and noise filtering have been successfully implemented using Simulink model and GUI of MATLAB.

\section{ACKNOWLEDGEMENTS}

The authors are thankful to Dr. Neeraj R. Prasad, Assistant Professor, Shivaji University, Kolhapur for critical suggestions while writing this research article. Similarly we are also thankful to our students Ms. Puja Nandimath, Ms. Saniya Kole and Ms. Ashwini Kamble for their all possible support during experimental work. Similarly we are thankful to all those who have supported either directly or indirectly in this research work.

\section{REFERENCES}

[1] Millard, A., (2004) "Playing with Poier-The Electric Guitar", A History of an American Icon, 123.

[2] Arnardottir, S., Abel, J. S., \& Smith III, J. O. (2008) "A digital model of the Echoplex tape delay", Audio Engineering Society Convention 125

[3] Persson, F., Augustsson, T., \& Andersson, A. P. (2012) "Effect tapper: interactive use of effects for musicians"

[4] Raffel, C., \& Smith, J. (2010, September) "Practical modeling of bucket-brigade device circuits", 13th International Conference on Digital Audio Effects (DAFx'10)

[5] Zhang, J., Nezan, J. F., \& Cousin, J. G. (2012, June) "Implementation of motion estimation based on heterogeneous parallel computing system with opencl", High performance computing and communication \& 2012 IEEE 9th International Conference on Embedded Software and Systems (HPCC-ICESS), 2012 IEEE 14th International Conference on (pp. 41-45)

[6] Jot, J. M., \& Chaigne, A. (1991) "Digital delay networks for designing artificial reverberator", Audio Engineering Society Convention 90

[7] Smith, S. W. (1997) The scientist and engineer's guide to digital signal processing.

[8] Mukhopadhyay, M., \& Ranjan, O. (2013) "An Intuitive Design Approach For Implementing Real Time Audio Effects"

[9] Hasnain, S. K., Daruwalla, A. D., \& Saleem, A. I. (2009) “A unified approach in Audio Signal Processing using the TMS320C6713 and Simulink Blocksets" Computer, Control and Communication, 2009 2nd International Conference on (pp. 1-5). IEEE.

[10] Gribonval, R., \& Bacry, E. (2003) "Harmonic decomposition of audio signals with matching pursuit". Signal Processing, IEEE Transactions on, 51(1), 101-111

[11] Reilly, A., \& McGrath, D. (1995) "Convolution processing for realistic reverberation" Audio Engineering Society Convention 98

[12] Bruns, C., \& Vahldieck, R. (2005) "A closer look at reverberation chambers-3-D simulation and experimental verification", Electromagnetic Compatibility, IEEE Transactions on, 47(3), 612-626.

[13] Sakamoto, N., Kurahashi, A., Edahiro, Y., Yamaguchi, S., \& Kogure, T. (1981), “A Digital Audio Reverberator", Audio Engineering Society Convention 70

[14] Spratt, K., \& Abel, J. S. (2008) "A digital reverberator modelled after the scattering of acoustic waves by trees in a forest", Audio Engineering Society Convention 125

[15] Murphy, D. T. (2006), "Archaeological acoustic space measurement for convolution reverberation and auralization applications", Proceedings of the 9th Int. Conference on Digital Audio Effects (DAFx-06), Montreal, Canada (pp. 18-20).

[16] Bode, H. (1984) "History of electronic sound modification", Journal of the Audio Engineering Society, 32(10), 730-739.

[17] Czubak, A., \& Raheja, G. (2007) “Guitar Effects Generator Using DSP”, Senior Project Proposal.

[18] Park, J. Y., Chang, J. H., \& Kim, Y. H. (2010) "Generation of independent bright zones for a twochannel private audio system", Journal of the Audio Engineering Society, 58(5), 382-393. 
[19] Byun, K., Kwon, Y. S., Koo, B. T., Eum, N. W., Jeong, K. H., \& Koo, J. E. (2009). "Implmentation of digital audio effect SoC", Multimedia and Expo, 2009. ICME 2009. IEEE International Conference on (pp. 1194-1197). IEEE.

[20] Singh, G., Savita, K., Yadav, S., \& Purwar, V. (2013) "Design Of Adaptive Noise Canceller Using LMS Algorithm”, International Journal of Advanced Technology \& Engineering Research (IJATER), 3(3), 85-89.

\section{Authors}

Yashwant V Joshi is Professor in Department of Electronics and Telecommunication Engineering, SGGS Institute of Engineering and Technology, Nanded. He has completed his Bachelor's degree (BE) and Master's degree in Electronics Engineering, from SGGS Institute of Engineering and Technology, Nanded in 1986 and 1991 respectively. He has completed doctoral degree in Electronics engineering in 1998 from IIT, Delhi. His areas of expertise include Digital Signal Processing, Image Processing, VLSI and Embedded System.

Saurabh R Prasad is working as Assistant Professor at DKTE Society's Textile and Engineering Institute, Ichalkaranji and also research scholar of Shivaji University, Kolhapur. He has about 12 years of teaching experience. He has expertise in the field of microwave engineering, audio and video engineering and digital signal processing.

Pawan K Gaikwad is Assistant Professor in Department of Electronics, Shivaji University, Kolhapur. He has completed M.Sc. in Electronics in 1999 from University of Pune and Ph D in 2010 from Shivaji University, Kolhapur. His areas of expertise include VLSI and Signal Processing. 